\author{
A.V. HAMZA \\ M.W. NEWMAN \\ P.A. THIELEN \\ H.W.H. LEE \\ T. SCHENKEL ${ }^{*}$ \\ J.W. MCDONALD \\ D.H. SCHNEIDER
}

\section{Exciton dispersion in silicon nanostructures formed by intense, ultra-fast electronic excitation}

University of California, Lawrence Livermore National Laboratory, Livermore, CA 94 551, USA

\begin{abstract}
Received: 2 October 2001/Accepted: 18 July 2002 Published online: 25 October 2002 • (C) Springer-Verlag 2002

ABSTRACT The intense, ultra-fast electronic excitation of clean silicon $(100)-(2 \times 1)$ surfaces leads to the formation of silicon nanostructures embedded in silicon, which photoluminesce in the yellow-green $(\sim 2-\mathrm{eV}$ band gap). The silicon surfaces were irradiated with slow, highly charged ions (e.g. $\mathrm{Xe}^{44+}$ and $\mathrm{Au}^{53+}$ ) to produce the ultra-fast electronic excitation. The observation of excitonic features in the luminescence from these nanostructures has recently been reported. In this paper we report the dispersion of the excitonic features with laser excitation energy. A phonon-scattering process is proposed to explain the observed dispersion.
\end{abstract}

PACS 71.35.-y; 63.20.Ls

1

\section{Introduction}

Bulk silicon is a poor light emitter owing to its indirect band gap. Since 1990 many authors have observed visible-light emission from silicon nanostructures. Two of the prominent examples are porous silicon (e.g. [1,2]) and silicon nanoclusters in silica (e.g. [3]). Visible emission has also been observed from one-dimensionally confined silicon in silicon $/ \mathrm{SiO}_{2}$ superlattices [4]. Many authors show that the visible emission can be due to quantum confinement of the excitation to a structure with less than 5-nm radius (Bohr radius of an exciton in silicon) (e.g. [2]). Confinement leads to a widening of the band gap and increased oscillator strength for the transition (e.g. [5]). The extent of changes (direct versus indirect) in the band structure of nanostructures of silicon is still a controversial topic (e.g. [6,7]).

Recently, we have briefly reported the observation of light emission from nanostructures on silicon formed by intense, ultra-fast electronic excitation [8]. The intense, ultra-fast electronic excitation of clean silicon $(100)-(2 \times 1)$ surfaces leads to the formation of silicon nanostructures embedded in silicon, which photoluminesce at $\sim 560$-nm wavelength $(\sim 2-\mathrm{eV}$ band gap). The silicon surfaces were irradiated with slow,

Fax: +1-925/423-7040, E-mail: Hamza1@1lnl.gov

* Present address: University of California, Lawrence Berkeley National Laboratory, Berkeley, CA 94 720, USA highly charged ions (e.g. $\mathrm{Xe}^{44+}$ and $\mathrm{Au}^{53+}$ ) to produce the electronic excitation. The observation of excitonic features in the luminescence is particularly unusual for silicon nanostructures. The temperature dependence of the luminescence and the measurement of the triplet-singlet splitting of the emission strongly support the excitonic assignment.

Intense, ultra-fast electronic excitation of a semiconductor surface such as silicon leads to the promotion of many $\left(>10^{3}\right)$ valence (bonding) electrons into the conduction bands (antibonding states). The promotion of a single electron or even many electrons into antibonding states does not impart a sufficient force for a sufficient length of time to cause displacement of the atomic nuclei. In contrast, the high density of electrons in antibonding states created by an intense, ultra-fast electronic excitation can impart an impulse leading to atomic displacements [9-12]. Direct evidence of atomic motion on $\mathrm{a} \sim 160$-fs time scale due to antibonding state excitation is reported by Petek et al. [13].

The deposition of potential energy from slow, highly charged ions (SHCI) such as $\mathrm{Xe}^{44+}$ (616-keV kinetic energy in this study) in semiconductor surfaces forms highly localized regions of intense electronic excitation $[10,14]$ on femtosecond time scales [15]. We define slow ions as ions with velocities less than the Bohr velocity. At these velocities the electrons in the solid surface can respond quickly to the motion of the incident ion, allowing for neutralization of the ion in femtoseconds. Slow, highly charged ions provide their electronic excitation in a nanometer-sized volume. The relaxation of the intense electronic excitation leads to motion of the atoms in the surface, which can freeze into metastable geometric structures with novel electronic properties. The novel electronic property produced in silicon $(100)-(2 \times 1)$ is photoluminescence (PL) in the yellow-green [8]. The emission was assigned to photogenerated excitons within the individual SHCI impact sites, based on the temperature dependence of the photoluminescence signal. Here, we report the dispersion of the excitonic photoluminescence observed from these nanometer-sized defects in silicon surfaces produced by slow, highly charged ions.

\section{$2 \quad$ Experimental}

Samples of float-zone silicon (100) were heated to $1300 \mathrm{~K}$ in ultra-high vacuum (base pressure $<5 \times 10^{-10}$ Torr) 
to produce a clean surface exhibiting a $(2 \times 1)$ low-energy electron-diffraction pattern. Secondary-ion mass spectroscopy with highly charged ions was performed to ensure complete removal of the native oxide layer and any surface contaminants. The samples were then irradiated at normal incidence with slow highly charged ions extracted from the electronbeam ion trap [16]. Secondary electrons emitted from individual ion impacts were detected with an annular microchannel plate to monitor the total ion dose with high efficiency $(\sim 100 \%)$ [17]. After exposure the samples were removed from vacuum and transferred to a second vacuum chamber for the photoluminescence experiments.

Photoluminescence measurements were performed using the doubled output from a femtosecond Ti-sapphire laser and a variable-emission optical parametric amplifier as the excitation source. The luminescence was collected using a scanning monochromator with a holographic grating. A photomultiplier tube was used for photon detection using a lock-in amplifier for signal processing. All samples were reintroduced into vacuum $\left(<10^{-3}\right.$ Torr $)$ prior to the photoluminescence measurements.

\section{Results and discussion}

Atomic force microscopy images revealed the formation of craters at individual slow, highly charged ionimpact sites (see Fig. 1). The craters were $\sim 15 \mathrm{~nm}$ in diameter for $\mathrm{Xe}^{44+}$. For $\mathrm{Xe}^{44+}$ impacts, the number of removed silicon atoms was $\sim 100 /$ ion as measured by catching the removed atoms on a diamond-like carbon surface, which was subsequently analyzed by heavy-ion backscattering (see [10] for a description of the technique). The photoluminescence signal from the impact sites is linearly proportional to the number of SHCI impinging on the surface (see Fig. 2). The PL signal increased linearly by a factor of $\sim 8$ with $\mathrm{Xe}^{40+}$ dose (irradiance) from $1 \times 10^{10} / \mathrm{cm}^{2}$ to $2 \times 10^{11} / \mathrm{cm}^{2}$. Above

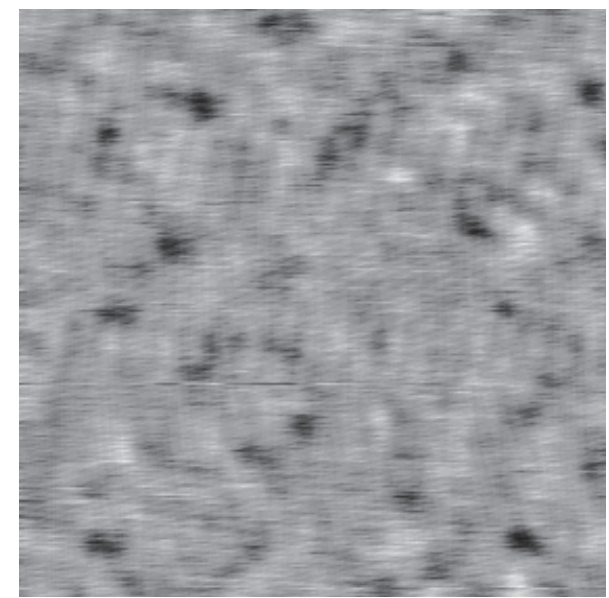

FIGURE 1 Atomic force micrograph of individual $\mathrm{Xe}^{44+}$ impact sites on $\mathrm{Si}(100)$. Float-zone $\mathrm{Si}$ irradiated with $616-\mathrm{keV} \mathrm{Xe}^{44+}$ (dose $\sim 10^{10}$ ions $/ \mathrm{cm}^{2}$ ). The imaged area is $500 \mathrm{~nm}$ by $500 \mathrm{~nm}$ and the individual impact craters are $\sim 15 \mathrm{~nm}$ in diameter

$2 \times 10^{11} / \mathrm{cm}^{2}$ to $1 \times 10^{12} / \mathrm{cm}^{2}$ the PL intensity increased only by a factor of $\sim 2$. A dose of $\sim 2 \times 10^{11} / \mathrm{cm}^{2}$ is the irradiance where overlap of impact sites begins, indicating that the impact sites have a diameter of $\sim 15 \mathrm{~nm}$, in agreement with the atomic force microscope measurements. The linear dependence of the PL intensity on dose also confirms that the PL comes from the individual impact sites and suggests that the $15-\mathrm{nm}$ craters are the emissive sites.

The features of the PL suggest that the emission results from the radiative recombination of a photogenerated exciton localized within the individual SHCI impact sites. The energy spacing of the luminescence peaks is that of a hydrogenic series given by $E_{\mathrm{N}}=E_{0}-\alpha / n$, where $n$ is the principal quantum number, $E_{0}$ is the direct interband transition, and $\alpha$ is the exciton binding energy. Only the $n=1$ and $n=2$ states are clearly resolved. The band-gap energy of this exciton is much higher
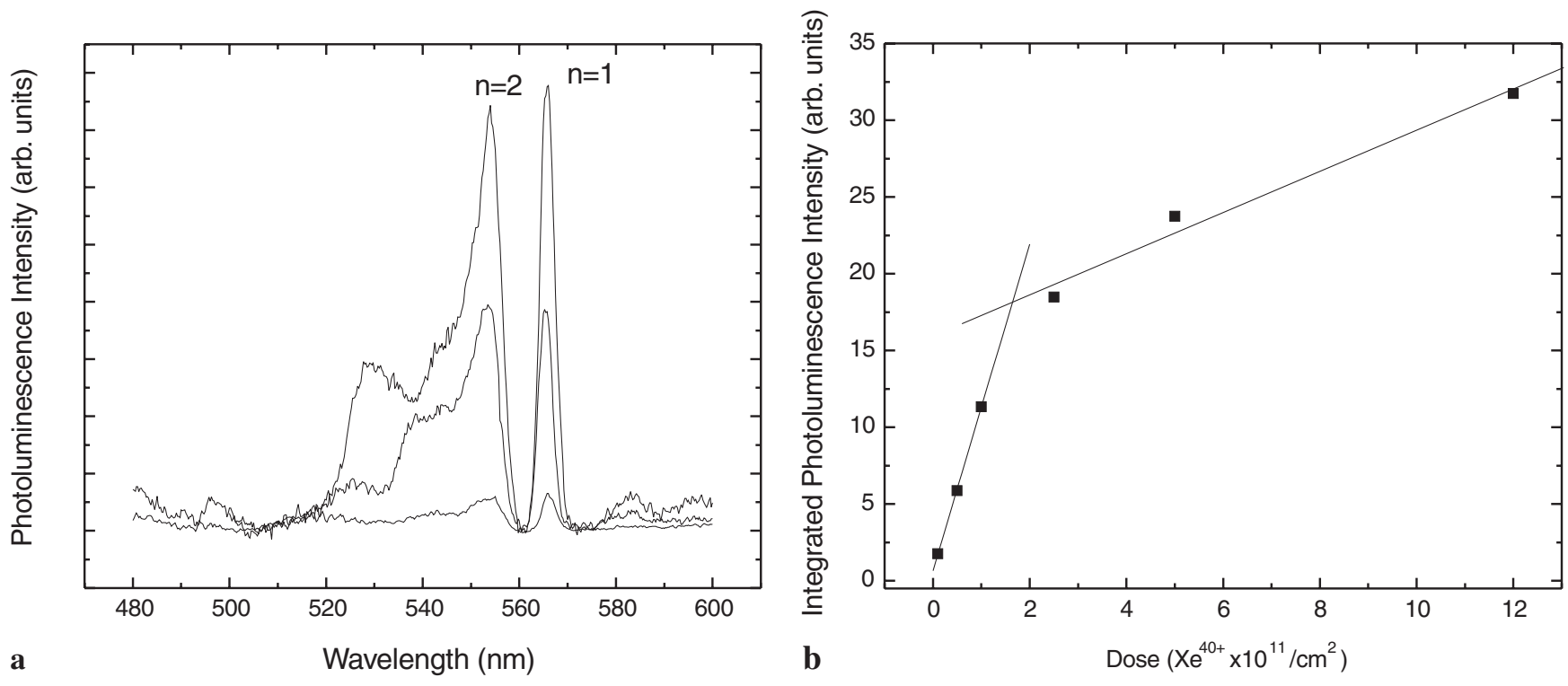

FIGURE 2 a Photoluminescence spectra from $\mathrm{Si}(100)$ samples irradiated with $1 \times 10^{10}, 1 \times 10^{11}$, and $5 \times 10^{11} \mathrm{Xe}^{40+}$ ions $/ \mathrm{cm}^{2}$. The photoexcitation wavelength was $400 \mathrm{~nm}$ for the spectra displayed. The exciton features that are well resolved are labeled for their principal quantum numbers, $n=1$ and $n=2$, respectively. b Plot of the increase in the integrated photoluminescence intensity for the $n=1$ exciton as a function of Xe ${ }^{40+}$-ion irradiance. The lines are least-squares fits to the low-dose data $\left(<2 \times 10^{11} \mathrm{Xe}^{40+}\right.$ ions $\left./ \mathrm{cm}^{2}\right)$ and the high-dose data $\left(\geq 2 \times 10^{11} \mathrm{Xe}^{40+}\right.$ ions $\left./ \mathrm{cm}^{2}\right)$ 
than that of bulk silicon. Thus, a barrier must exist that keeps the exciton confined to the higher band-gap region. Since the samples are removed from vacuum and reintroduced to vacuum for PL measurements, the native oxide of silicon may provide such a barrier.

Upon changing the excitation energy in the photoluminescence measurement, the energy of the exciton shifts. Figure $3 \mathrm{a}$ shows a few of the photoluminescence spectra for various laser excitation energies from a $\mathrm{Si}(100)$ sample irradiated with $742-\mathrm{keV}$ (kinetic energy) $\mathrm{Au}^{53+}$ ions. Figure $3 \mathrm{~b}$ shows the energy loss (excitation energy minus emitted photon energy) for the $n=1$ and $n=2$ exciton transitions versus laser excitation energy for the same sample. The photoluminescence from the $\mathrm{Au}^{53+}$-irradiated sample was identical to the $\mathrm{Xe}^{44+}$. irradiated sample except for the increased intensity with increased charge (see Fig. 4). Only the irradiated portions of the surface exhibited luminescence. Throughout the range of excitation energies used in this investigation the photoluminescence signal maintained the same excitonic structure.

No effect on the photoluminescence signal was observed with variation of the kinetic energy of the ions from 50 to $800 \mathrm{keV}$. As shown in Fig. 4a threshold was observed for the luminescence. Ions with potential energy less than $\sim 10 \mathrm{keV} /$ ion $\left(\mathrm{Xe}^{23+}\right)$ produced no photoluminescence signal. The photoluminescence signal was linearly proportional to the potential energy carried by the ion up to $\sim 130 \mathrm{keV} /$ ion $\left(\mathrm{Au}^{63+}\right)$ once the $\sim 10-\mathrm{keV} /$ ion threshold was reached.

If the energy of the PL above the bulk silicon band gap is due to confinement of the exciton in the nanostructure, it would be expected that the emission wavelength should shift with the dimension of the nanostructure [18]. The PL intensity increase with potential energy of the ion at constant irradiance suggests that the area of each impact increases with potential energy of the ion. This idea is consistent with the observation of increasing sputter yield of GaAs with potential energy

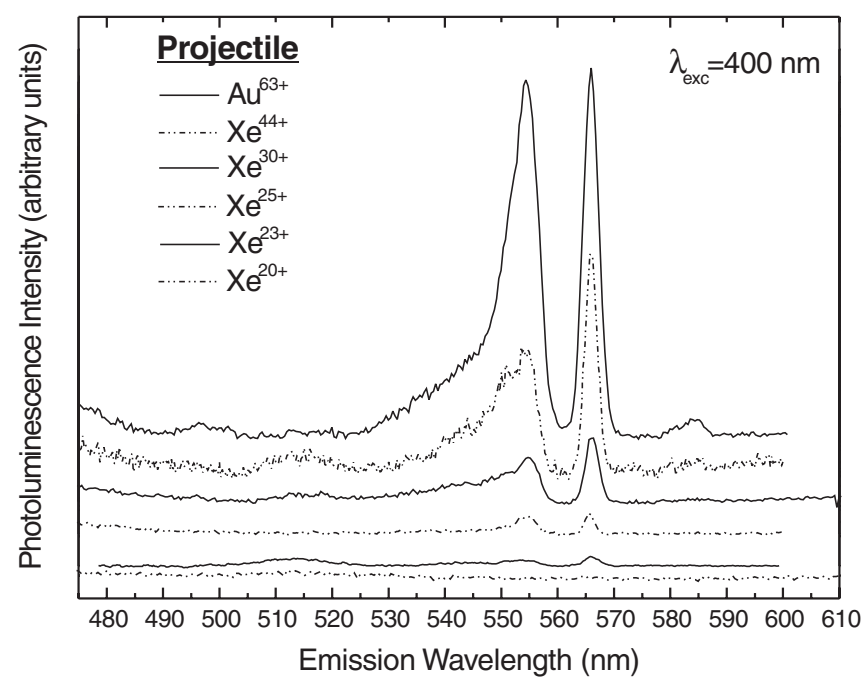

FIGURE 4 Photoluminescence spectra for samples irradiated with the labeled ion at a constant irradiance of $1 \times 10^{11}$ ions $/ \mathrm{cm}^{2}$. Threshold for the observed luminescence is $\mathrm{Xe}^{23+}, \sim 10-\mathrm{keV}$ potential energy/ion

for highly charged ions [10]. That is, if the sputter yield increases with potential energy, the crater size should increase with potential energy. No shift in the PL is observed with potential energy. In addition, the lateral dimensions of the nanostructures are too large (greater than the $\sim 5-\mathrm{nm}$ Bohr radius for excitons in bulk silicon [19]) for strong confinement. If confinement plays a role in the observed emission, the confinement dimension is normal to the surface (as in the $\mathrm{Si} / \mathrm{SiO}_{2}$ superlattice [4]) and unchanged by an increase in the potential energy of the ion. An alternative explanation for the emission observed, that cannot be ruled out, is that the slow, highly charged ion impact induces a phase transformation in the impact volume to a structure (e.g. $\beta$-Sn) with a direct band gap.
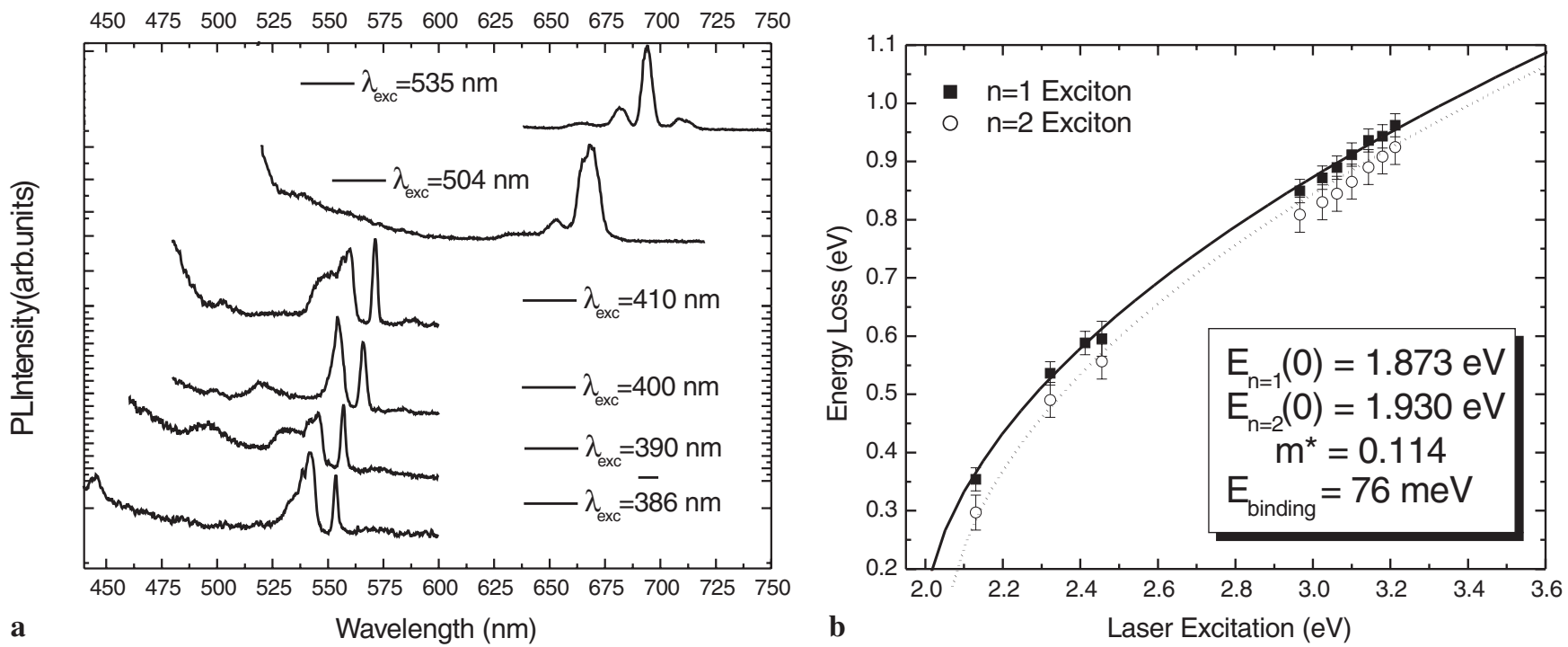

FIGURE 3 a Dispersion of the exciton features as a function of photoexcitation wavelength from $386 \mathrm{~nm}$ to $535 \mathrm{~nm}$. b Plot of energy loss (photoexcitation energy minus the energy of the luminescence photon) versus the laser excitation energy. The filled symbols represent the data for the $n=1$ exciton and the open symbols represent the data for the $n=2$ exciton. Lines are fits to (4), with the solid line for the $n=1$ exciton and the dashed line for the $n=2$ exciton. The parameters for the fits are displayed in the inset 
The luminescence spectra show dispersion in the visible region. This implies that the photoluminescence does not originate from annihilation of an exciton in its lowestmomentum state, $E_{n}(\boldsymbol{k}=0)$, where $E_{n}$ is the energy of the exciton in principal quantum state $n$ and $\boldsymbol{k}$ is the exciton momentum. For recombination from the $E_{n}(0)$ state, no shift in the luminescence peak is expected with excitation energy. Recombination from $\boldsymbol{k}=0$ would appear as a straight line with slope 1 in the energy-loss plot of Fig. 3b. Alternatively, for Raman-scattering events the energy difference between the incident photon and the scattered light remains constant. Neither of these two scenarios is supported by the plot in Fig. 3b. In addition, the magnitude of the energy loss is quite large, more than typical phonon energies $(<200 \mathrm{meV})$.

A similar dispersive behavior to that observed here has been reported for the yellow and green excitonic series in $\mathrm{Cu}_{2} \mathrm{O}$ by $\mathrm{Yu}$ and Chen [20] and $\mathrm{Yu}$ and Cardona [21]. They considered a three-phonon scattering process involving two dispersion-less longitudinal optical (LO) phonons and a single dispersive longitudinal acoustic (LA) phonon to explain the exciton dispersion. Figure 5 shows a diagram of the excitation and relaxation processes involved which lead to the dispersion of the exciton in the luminescence spectra. Since an exciton is a real excitation it must obey conservation of energy and momentum laws. An incident photon with energy $\hbar \omega_{1}$ will cause an electron from the highest point in the valence band with electron momentum equal to zero to make a vertical transition to a state in the exciton band. We assume a parabolic shape to the exciton band for simplicity. In order to populate a real state the emission of a phonon with wavevector $\boldsymbol{k}_{1}$ is required for momentum conservation, to reach the exciton band. The drawing in Fig. 5 shows a direct band gap, the exciton minimum directly above the valence-band maximum, for simplicity, but this is not necessary for the description presented here. Since the frequency involved in scattering a visible photon is large we assume that this phonon is an optical phonon. It is well known that excitons are more likely to be scattered by longitudinal phonons [22]. In addition we can approximate the longitudinal optical phonon to be dispersion-less.

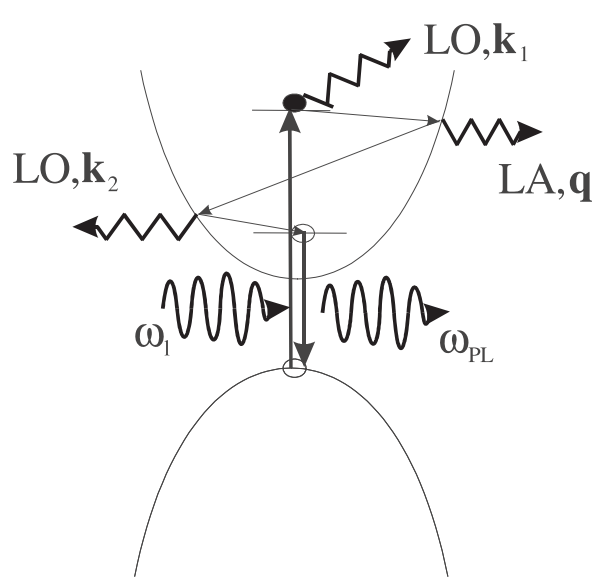

FIGURE 5 The excitation and relaxation process for a photoexcited exciton that undergoes phonon scattering to reduce its energy. The scattering process involves two dispersion-less LO phonons and a single dispersive LA phonon
The energy and wavevector of the photoexcited exciton are determined by energy conservation [21]:

$$
E_{n}\left(\boldsymbol{k}_{1}\right)=E_{n}(0)+\hbar^{2} k_{1}^{2} / 2 M=\hbar\left(\omega_{1}-\omega_{0}\right),
$$

where $M=\left(m_{\mathrm{e}}^{*}+m_{\mathrm{h}}^{*}\right)$ is the effective mass of the exciton and $\omega_{1}$ and $\omega_{0}$ are the energies of the incident photon and the LO phonon, respectively. Notice that while energy conservation is in general not necessary for transitions to virtual intermediate states during Raman scattering, in this case it is necessary because of the real excitation of the $n=1$ exciton.

Upon reaching the exciton state, the excitation will 'cool' through the emission of a single dispersive longitudinal acoustic phonon with wavevector $\boldsymbol{q}$, which we assume to have a linear dispersion relation, $\omega_{\mathrm{LA}}=v_{\mathrm{LA}}|\boldsymbol{q}|$, where $v_{\mathrm{LA}}$ is the speed of the LA phonons. The exciton will then undergo a final scattering off a second LO phonon with wavevector $\boldsymbol{k}_{2}$, into a state from which the electron-hole pair will recombine with the emission of a photon with energy $\hbar \omega_{\mathrm{PL}}$.

From Fig. 5 we see that conservation of energy and momentum are given respectively as:

$\hbar \omega_{1}=2 \hbar \omega_{0}+\hbar \omega_{\mathrm{LA}}+\hbar \omega_{\mathrm{PL}}$

$q=\boldsymbol{k}_{1}-\boldsymbol{k}_{2}$

where $\hbar \omega_{\mathrm{PL}}$ is the energy of the luminescence photon. Additionally, by combining the two conservation laws and the dispersion relation for the LA phonon and the energy of the exciton (1) an expression for the exciton dispersion is obtained and given by:

$$
\Delta E=2\left(\hbar \omega_{0}-M v_{\mathrm{LA}}^{2}\right)+\left[8 M \nu_{\mathrm{LA}}^{2}\left(\hbar \omega_{1}-\hbar \omega_{0}+E_{n}(0)\right)\right]^{0.5}
$$

Therefore, we see that from (4) the energy loss $(\Delta E=$ $\left.\hbar \omega_{1}-\hbar \omega_{\mathrm{PL}}\right)$ varies as $\left[\hbar \omega_{1}-\hbar \omega_{0}+E_{n}(0)\right]^{0.5}$.

The lines in Fig. $3 \mathrm{~b}$ are fits to (4) for the $n=1$ and $n=2$ excitons with two free parameters, $M$ and $E_{n}(0)$. For this calculation we assume $\nu_{\mathrm{LA}}=v_{\mathrm{La}}^{\text {bulk } \mathrm{Si}}$ and $\omega_{0}=\omega_{0}^{\text {bulk } \mathrm{Si}(\mathrm{LO})}$. Since the speed of sound is principally dependent on the atomic density, the structural reorganized region (i.e. the single slow highly charged ion defect) will have a similar atomic number density to bulk silicon. The second assumption is of little consequence since contributions from these terms are small compared to $E_{n}(0)$ and $M v_{\mathrm{LA}}^{2}$. The parametric fits for the $n=1$ and $n=2$ exciton dispersion yield $M=0.114 \pm$ $0.005 m_{\mathrm{e}}, E_{n=1}(0)=1.87 \pm 0.02 \mathrm{eV}$, and $E_{n=2}(0)=1.90 \pm$ $0.02 \mathrm{eV}$. Using the parameters given above, the fit to the data is quite good.

\section{$4 \quad$ Conclusions}

The intense, ultra-fast electronic excitation that results from the deposition of potential energy from slow, highly charged ion impacts on silicon (100) surfaces leads to the formation of nanometer-sized structures. Photogenerated excitons within these regions radiatively decay, emitting $\sim 2-\mathrm{eV}$ light. The exciton dispersion evidenced by the square-root dependence of the energy loss (the laser excitation energy less the luminescence energy) on the laser excitation energy is explained by a cooling process involving an acoustic phonon. 
ACKNOWLEDGEMENTS This work was performed under the auspices of the U.S. Department of Energy by the University of California, Lawrence Livermore National Laboratory, under Contract No. W-7405ENG-48 and was supported by the Division of Chemical Sciences of the Office of Science.

\section{REFERENCES}

1 L.T. Canham: Appl. Phys. Lett. 57, 1046 (1990)

2 P.D.J. Calcott, K.J. Nash, L.T. Kane, D. Brumhead: J. Phys: Condens. Matter 5, L91 (1993)

3 M.L. Brongersma, P.G. Kik, A. Polman, K.S. Min, H.A. Atwater: Appl. Phys. Lett. 76, 351 (2000)

4 D.J. Lockwood, Z.H. Lu, J.-M. Baribeau: Phys. Rev. Lett. 76, 539 (1996)

5 B. Delley, E.F. Steigmeier: Phys. Rev. B 47, 1397 (1993)

6 A. Nakajima, Y. Sugita, K. Kawamura, H. Tomita, N. Yokoyama: J. Appl. Phys. 80, 4006 (1996)

7 Z.X. Ma, X.B. Liao, G.L. Kong, J.H. Chu: Appl. Phys. Lett. 75, 1857 (1999)

8 A.V. Hamza, M.W. Newman, P.A. Thielen, H.W.H. Lee, T. Schenkel, J.W. McDonald, D.H. Schneider: Appl. Phys. Lett. 79, 2973 (2001)
9 P. Stampfli: Nucl. Instrum. Methods B 107, 138 (1996); P. Stampfli, K.H. Bennemann: Phys. Rev. B 49, 7299 (1994)

10 T. Schenkel, A.V. Hamza, A.V. Barnes, D.H. Schneider, J.C. Banks, B.L. Doyle: Phys. Rev. Lett. 81, 2590 (1998)

11 H.W.K. Tom, G.D. Aumiller, C.H. Britocruz: Phys. Rev. Lett. 60, 1438 (1988)

12 L. Huang, J.P. Callan, E.N. Glezer, E. Mazur: Phys. Rev. Lett. 80, 185 (1998)

13 H. Petek, M.J. Weida, H. Nagano, S. Ogawa: Science 288, 1402 (2000)

14 T. Schenkel, A.V. Barnes, T.R. Niedermayr, M. Hattass, M.W. Newman, G.A. Machicoane, J.W. McDonald, A.V. Hamza, D.H. Schneider: Phys. Rev. Lett. 83, 4273 (1999)

15 M. Hattass, T. Schenkel, A.V. Hamza, A.V. Barnes, M.W. Newman, J.W. McDonald, T.R. Niedermayr, G.A. Machicoane, D.H. Schneider: Phys. Rev. Lett. 82, 4795 (1999)

16 D.H. Schneider, M.A. Briere: Phys. Scr. 53, 228 (1996)

17 T. Schenkel, A.V. Barnes, A.V. Hamza, D.H. Schneider: Eur. Phys. J. D 1, 297 (1998)

18 T. van Buuren, L.N. Dinh, L.L. Chase, W.J. Siekhaus, L.J. Terminello: Phys. Rev. Lett. 80, 3803 (1998)

19 D.J. Lockwood: Solid State Commun. 92, 101 (1994)

20 P. Yu, Y.R. Shen: Phys. Rev. B 12, 1377 (1975)

21 P.Y. Yu, M. Cardona: Fundamentals of Semiconductors: Physics and Materials Properties (Springer, Berlin, Heidelberg 1999) pp. 403-406

22 Y. Toyozawa: Prog. Theor. Phys. 20, 53 (1958) 